\title{
Real-time two-photon confocal microscopy using a femtosecond, amplified Ti:sapphire system
}

\author{
G. J. BRAKENHOFF,* J. SQUIER, $\dagger$ T. NORRIS, $\dagger$ A. C. BLITON, $\ddagger$ M. H. WADE $\ddagger \&$ B. ATHEY§ \\ *Confocal Microscopy Group, Department of Molecular Cell Biology, University of Amsterdam, Pl. \\ Muidergr. 14, 1018 TV, Amsterdam, The Netherlands \\ "'Centre for Ultrafast Optical Science, University of Michigan, 2200 Bonisteel, IST Bldg RM \\ 1006, Ann Arbor, MI 48109-2099, U.S.A. \\ Meridian Instruments, Inc., 2310 Science Parkway, Okemos, MI 48864, U.S.A. \\ \#\#Department of Anatomy and Cell Biology, University of Michigan, Medical School, Ann \\ Arbor, MI 48109-0616, U.S.A.
}

Key words. Bilateral, confocal, real-time imaging, sectioning, three-dimensional imaging, TPA, two-photon fluorescence.

\begin{abstract}
Summary
The bilateral imaging approach known from confocal applications operating in the line mode was used to realize real-time two-photon imaging. It is shown that the sectioning inherent to two-photon imaging could be improved by the introduction of a confocal line aperture in the imaging path. Using a high-power, low-repetition-rate amplified Ti:sapphire system, various biological objects were visualized including live boar sperm.
\end{abstract}

\section{Introduction}

There are at present three ways to obtain two-dimensional optical sections from a three-dimensional specimen. These are: (a) by deconvolution of a series of conventional microscope images collected at various depths in the specimen (Agart et al., 1989); (b) with confocal imaging (Wilson \& Sheppard, 1984; Brakenhoff et al., 1985); (c) by the recently developed technique of two-photon fluorescence imaging (Denk et al., 1990). In the last of these, a fluorescence molecule is excited by simultaneous absorption of two photons of half the energy (i.e. twice the wavelength) of the corresponding single-photon excitation. The twophoton absorption depends on the probability of the simultaneous presence of two photons in the absorption volume and will thus be proportional to the square of the instantaneous two-photon excitation intensity (Denk et al., 1990). Thus the efficiency of excitation by two-photon absorption (TPA) will be enhanced by concentrating the laser excitation power in time into short pulses (see below). The power is also concentrated in space by focusing the laser beam to a point in the sample with a high-NA objective. The square power dependence then also causes the TPA fluorescence generation to take place most efficiently at the location of highest intensity, i.e. at the focal plane. This is the physical reason for the sectioning property of this type of imaging. The higher the NA of the lens, the steeper the axial gradients in the light distribution around the focus and the better the sectioning. The TPA sectioning can be improved by the introduction of an additional confocal aperture (Sheppard \& Gu, 1990). The real-time TPA imaging of biological specimens described in this paper was realized under the latter condition.

The reasons for using TPA excitation for the study of biological specimens are the indicated inherent sectioning, and the fact that with two-photon excitation bleaching of the specimen takes place only in the specimen plane where the TPA fluorescence generation occurs. This is in contrast with single-photon excitation microscopy where the specimen is bleached over its full depth. Also, the longer wavelength of the exciting radiation (compared with the one-photon case) allows better penetration of the exciting radiation in the specimen and a reduced damage sensitivity of biological specimens is expected (Denk et al., 1990). For these reasons it seems of interest to extend this technique to real-time observations. In particular, the absence of outof-focus bleaching and the reduced biological damage suggests more favourable conditions for the observation of (live) biological specimens for extended periods than with single-photon fluorescence imaging. Although not of relevance to the present work, TPA excitation at wavelengths that would require quartz-optics microscopes, i.e. use wavelengths below $350 \mathrm{~nm}$, may be possible with twophoton excitation at $500-700 \mathrm{~nm}$ using regular visible light optics. 


\section{Physics of two-photon fluorescence generation}

While TPA fluorescence microscopy has the advantages indicated above, there is the difficulty that very high power levels in the excitation beams are necessary because of the very low TPA cross-sections for the commonly used dyes (e.g. $10^{-58} \mathrm{~m}^{4} \mathrm{~s}^{-1}$ for fluoresceine, Denk et al., 1990). This makes it important to apply the pulsed TPA excitation in such a way that the fluorescence produced is maximized for a given permissible average excitation power. Because of the small TPA cross-sections, the excitation power limits will generally be determined by the non-TPA, i.e. single-photon interaction processes of the radiation with the specimen, and thus will be linearly proportional with the excitation intensity. Because of the long, typically infra-red, wavelengths used in TPA, the interaction can be expected to be mostly through direct absorption, leading to a temperature rise of the TPA excited volume. The specimen loading limited by the permissible temperature rise will then determine the maximum TPA excitation energy $E$ that can be applied to this volume. In view of the relaxation times for the vibrational levels (approximately $10^{-12} \mathrm{~s}$ ), this temperature rise will be virtually instantaneous in comparison with the thermal relaxation time $t_{\text {th }}$ of the TPA excited volume (see below).

If for the moment we assume pulses with an excitation power $P$ and duration of $t_{\mathrm{p}}$, i.e. an energy $E=t_{\mathrm{p}} \cdot P$ then, as indicated above, the TPA fluorescence generated in the specimen by one pulse of energy $E$ will be proportional to $E^{2}$. The key element of this discussion is now: if this energy $E$ would be distributed over $N$ pulses within $t_{\mathrm{th}}$, then each of those pulses with energy $E / N$ would generate a TPA fluorescence proportional to $(E / N)^{2}$, producing a total fluorescence within $t_{\text {th }}$ of $N \cdot(E / N)^{2}=E^{2} / N$, i.e. $1 / N$ times the amount of fluorescence that would be generated with this energy applied in one pulse.

Optimization of the TPA fluorescence generation for a certain permissible excitation power would thus require pulse repetition rates of the exciting laser system of the order of $1 / t_{\text {th }}$. The focal volumes of typical high-NA optics will be of the order of $0 \cdot 1$ to several cubic micrometres with thermal relaxation times ranging from $0 \cdot 1$ to a few microseconds, depending on focusing and imaging conditions (see Appendix A). This implies that pulse repetition rates in view of thermal loading should be of the order of a few hundred kilohertz up to $5 \mathrm{MHz}$. The latter value is applicable under optimal imaging conditions where the smallest optically defined excitation volumes can be realized. This is substantially lower than the operating frequencies of $76-100 \mathrm{MHz}$ of regular Ti:sapphire laser systems as used for instance by Denk et al. (1990) during earlier TPA work. In the Discussion and Appendix B we examine the specific excitation and specimen loading conditions in line and point systems.

\section{Real-time TPA imaging with amplified Ti:sapphire systems and bilateral scanning}

For realizing real-time TPA fluorescence imaging we have employed the bilateral scanning principle (Fig. 1) with cursor illumination and slit detection, as previously employed for confocal microscopy (Brakenhoff \& Visscher, 1992). This bilateral imaging approach has the property that the TPA fluorescence image can be created directly in a 2-D detection plane where it can either be recorded on a 2-D detector like a (cooled) CCD, photographic plate or-if sufficiently high image repetition rates can be realized-directly observed by eye. In the TPA work published by Denk et al. (1990) the excitation is point by point with the fluorescence detected with a photomultiplier and the TPA image generated at a later time from the data stored in computer memory. The bilateral unit used was a modified Insight Plus unit (Meridian Instruments, Okemos, MI, U.S.A.) mounted on an Nikon microscope (Optiphot-2). It was operating in the line illumination/detection mode where each scan of the bilateral scanning mirror generates a full confocal or TPA image (see Brakenhoff \& Visscher, 1992, for further details).

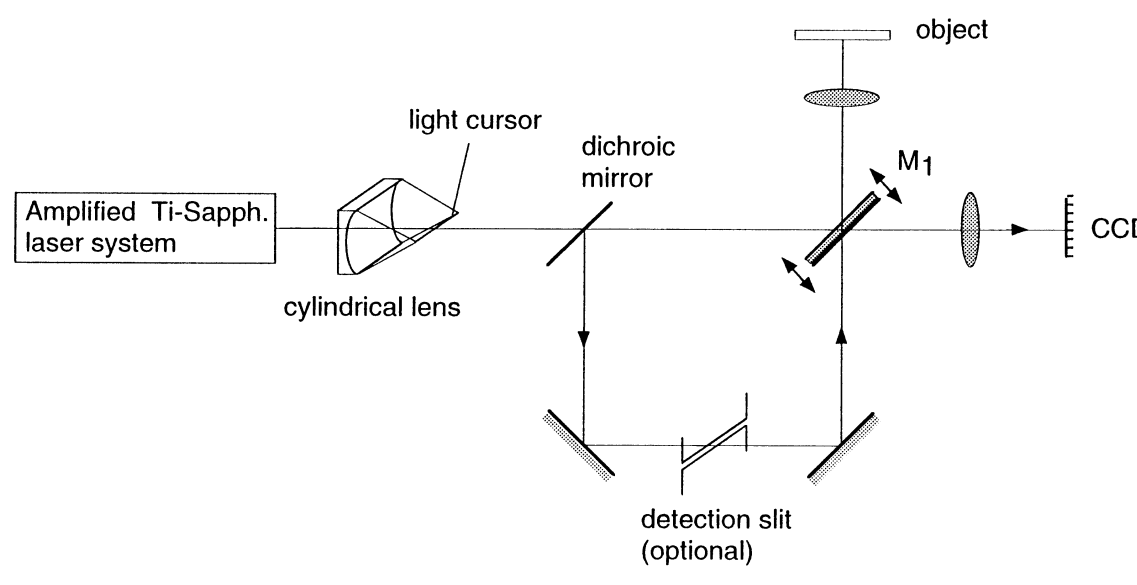

Fig. 1. TPA fluorescence generation with bilateral scanning and CCD detection. A line illumination pattern created by the cylindrical lens is scanned over the specimen by reflection of the front side of the bilateral mirror $\mathrm{M}_{1}$. The generated TPA fluorescence light is de-scanned by reflection on the same mirror surface and is led back and guided by reflection off the back side of $\mathrm{M}_{1}$ and a dichroic mirror with suitable reflection characteristics to a location on the CCD corresponding to the addressed specimen location. The detection slit required for sectioning in confocal imaging is not necessary with TPA, but can be used to improve the suppression of out-of-focus contributions. 
To satisfy TPA excitation conditions along an excitation line (cursor), substantially higher pulse power levels are required compared with point TPA as previously used by Denk et al. (1990). These pulse powers should actually be higher in comparison with the point mode by the ratio of illuminated line length over point dimension. Pulses of the required power are available from femtosecond, amplified Ti:sapphire systems such as the one developed by Rhee et al. (1994) which was used for the present experiments. This laser has the following properties: maximum pulse energy $200 \mathrm{~nJ}$, pulse duration $t_{\mathrm{p}}$ of $100 \mathrm{fs}$, pulse repetition rate of $250 \mathrm{kHz}$ and maximum average power of about $50 \mathrm{~mW}$. The operating wavelength during the experiments reported was between 780 and $810 \mathrm{~nm}$. The pulse repetition rate of $250 \mathrm{kHz}$ is in the proper range for matching the qua-thermal relaxation-optimal two photon generating conditions, as indicated above. At the same time this pulse repetition rate is far above the one necessary for real-time imaging in the line mode considering that with each pulse a line in the image can be generated, and only a $30-\mathrm{kHz}$ line repetition rate is needed to produce real time images at 30 images $\mathrm{s}^{-1}$ with 500-1000 lines required per image.

\section{Experiments and results}

Using the set-up indicated in Fig. 1 and the amplified Ti:sapphire system described, we first checked whether the characteristic TPA quadratic dependence on intensity could be observed in our line illumination/detection system. Figure 2 shows that was indeed the case.

The axial sectioning capability of TPA fluorescence generation has been documented before (Denk et al., 1990) and is one of the main reasons for using two photon microscopy. We have measured the sectioning power of our line system using a thin fluorescent ink layer as an object (Fig. 3), without and-to enhance the sectioning-with a confocal line aperture present in the imaging path. The confocal line aperture had a width equivalent to about twice the diffraction-limited resolution of the lens system used. As expected (Deitch et al., 1994), we observed a substantial improvement in the sectioning capabilities with the line aperture in place. Because of this improvement we decided to collect the data for real-time imaging shown below under this latter condition.

The sectioning and 3-D imaging capabilities of the system are demonstrated on a fixed fluorescent specimen consisting of pollen mixed with $4 \cdot 7-\mu \mathrm{m}$ beads (Fig. 4). These images are recorded on a video-type CCD (Pulnix integrating TM-745) with delayed read-out capabilities. Delaying the read-out permits the accumulation on the CCD of the detected photon fluxes for times up to a few seconds, improving the signal-tonoise ratio in the final collected image. As can be seen, good quality 3-D fluorescent images can be obtained with TPA line excitation.

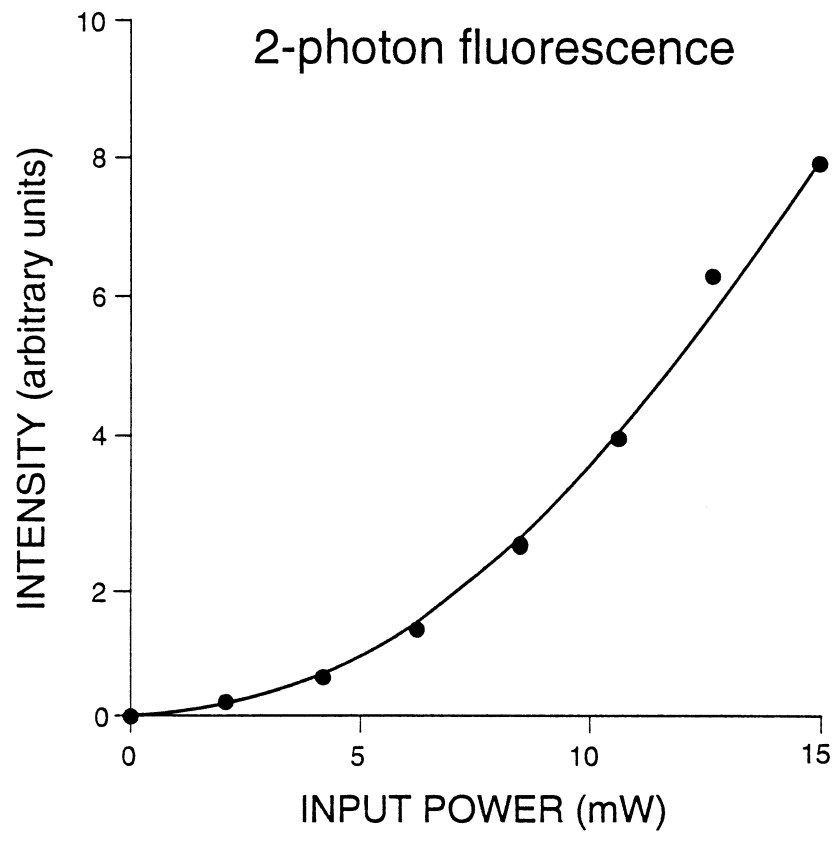

Fig. 2. Fluorescence intensity generated in a uniformly labelled fluorescent block specimen as a function of laser power input into the microscope objective. The filled circles are the measured points and the continuous line is a second-order polynomial fit through the data, illustrating the quadratic dependence expected in two-photon fluorescence excitation.

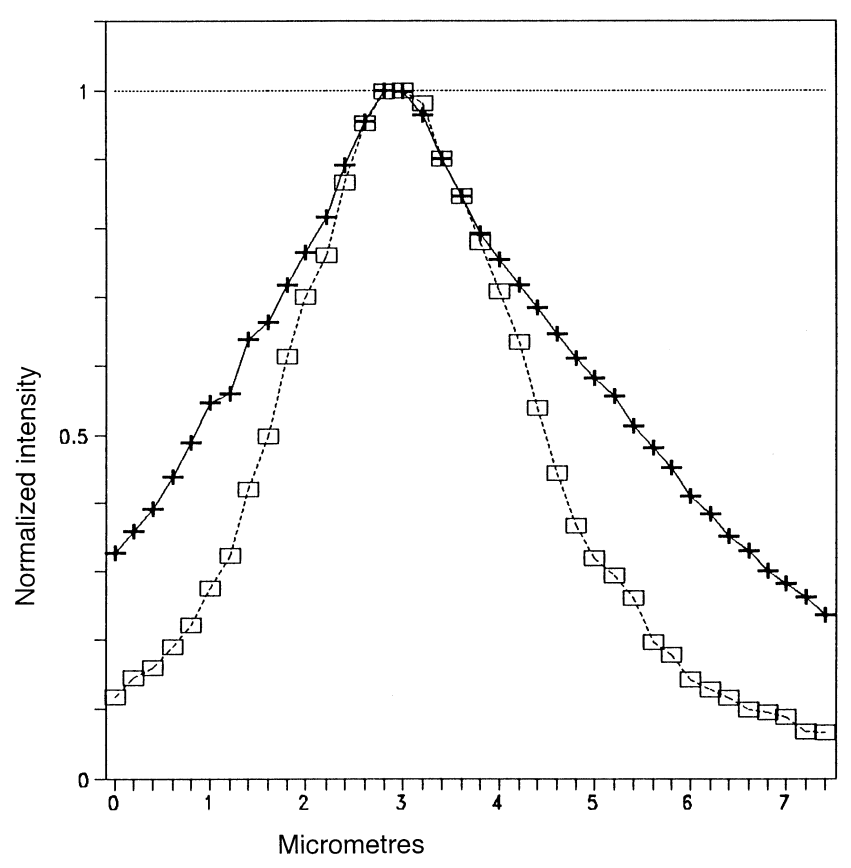

Fig. 3. The two-photon axial response from a thin fluorescent plane object, with line illumination, measured with a high-NA $(=1 \cdot 3)$ oilimmersion objective under imaging conditions without and with confocal slit aperture, showing a clear improvement in the latter case. (—) Two-photon Z-response; (- - - -) with confocal slit; (. . ...) one-photon Z-response. 
(a)

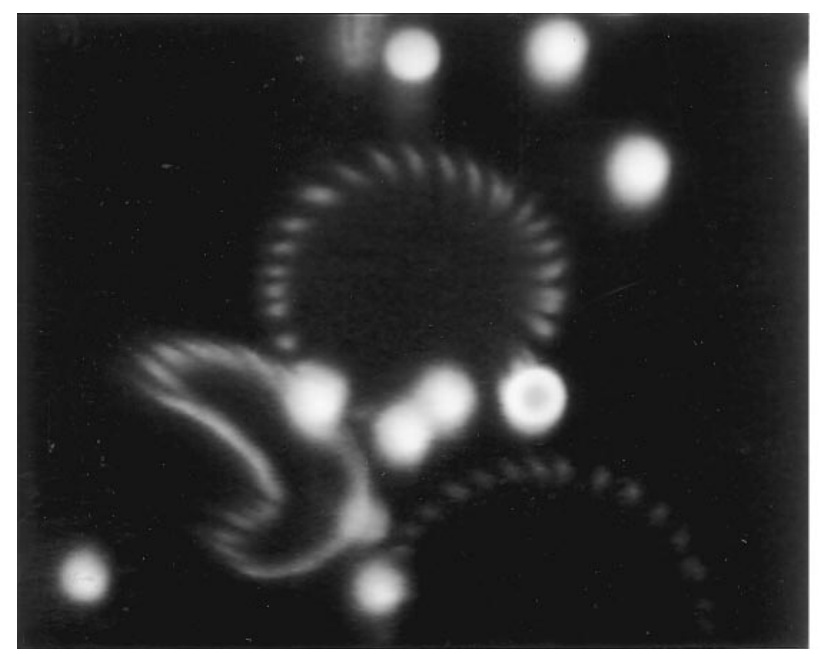

(b)

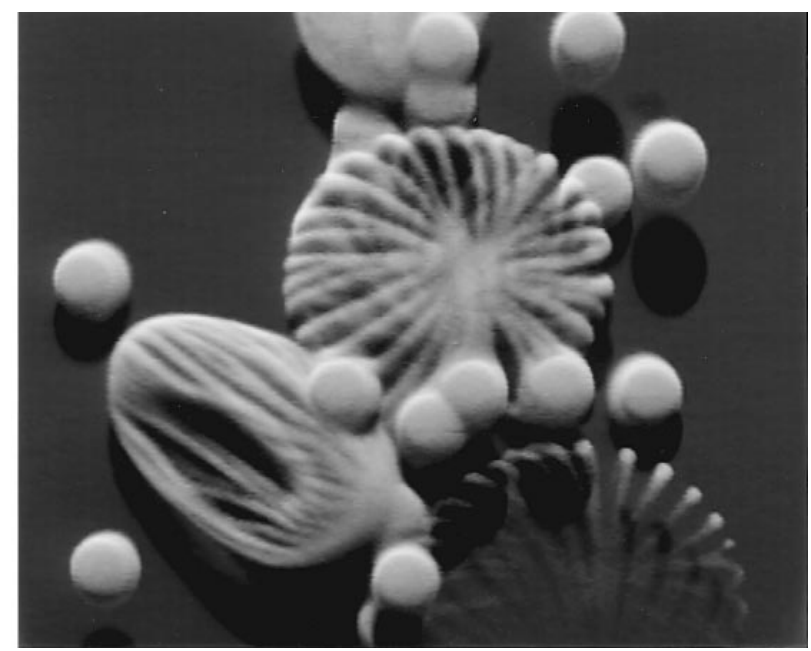

Fig. 4. (a) A TPA fluorescence image of a section of a specimen containing acridine-orange-labelled Spathiphyllum pollen mixed with phycoerythrin-labelled $4 \cdot 7-\mu \mathrm{m}$ beads with the confocally enhanced sectioning (see text). (b) A 3-D image of this specimen created from a series of sections, one of which is shown in (a). The rendering algorithm is the fluorescence simulation process as described by van der Voort $e t$ al. (1989) and modified by Hallgren \& Bucholz (1992).

Real-time TPA images (Fig. 5) of $2 \mu \mathrm{M}$ Indo-1AM-labelled live boar sperm were collected at intervals of $1 / 30 \mathrm{~s}$ with two-photon excitation at $780 \mathrm{~nm}$. Owing to the bilateral imaging approach (Brakenhoff \& Visscher, 1993), the sperm images could be both observed directly by eye and recorded with the CCD/video camera. The sperm bodies were observed alive and moving under TPA observation for up to $2 \cdot 5 \mathrm{~min}$. The limiting factor was in fact the bleaching of the dyes used.

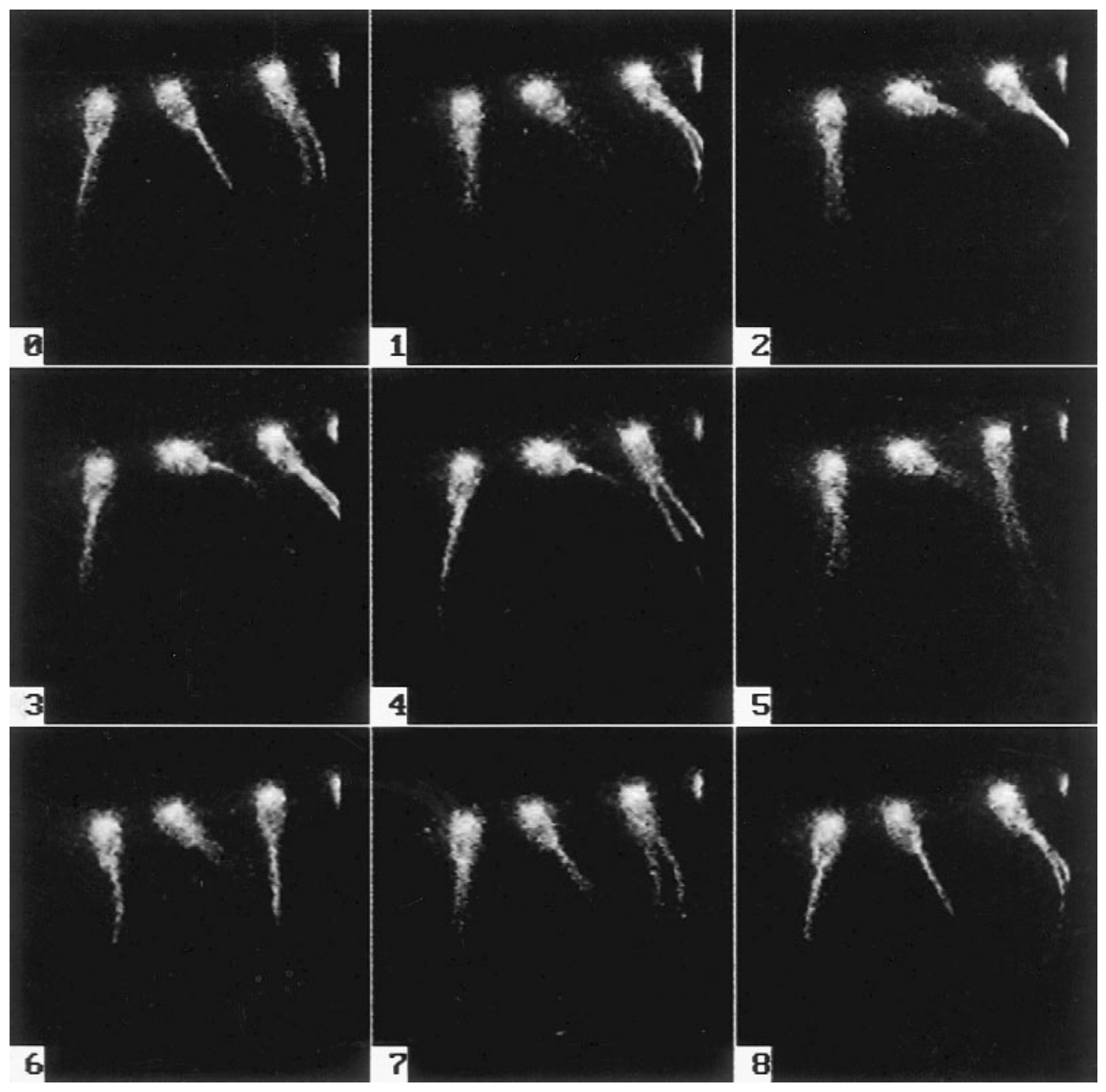

Fig. 5. Video-rate TPA imaging of live boar sperm cells labelled with $2 \mu \mathrm{M}$ INDO-1AM. The images were recorded with a intensified video system at $30 \mathrm{~Hz}$ image repetition rate for a duration of $2.5 \mathrm{~min}$ in total before bleaching reduced the image intensity to an unworkable intensity. Up to 4000 frames were collected. The images shown were collected sequentially at intervals of $1 / 30 \mathrm{~s}$ after about $1 \mathrm{~min}$ of illumination. The sperm cells, and especially the tails, show substantial lateral and axial movement from frame to frame with the axial movement, resulting in an occasional 'disappearance' of the tail due to the sectioning property of the imaging. 


\section{Discussion and conclusions}

We have demonstrated real-time TPA fluorescence imaging using an amplified Ti:sapphire laser system in combination with line bilateral scanning. This has in part been made possible by a felicitous interplay of pulse repetition rate and cursor excitation, as can be realized from a comparison of TPA fluorescence excitation in point and line systems. In Appendix B it is indicated first that in our line system, in comparison with Denk's (1990) point scanning system, for equal average TPA input power equal fluorescence output is generated. During the real-time TPA imaging of the boar sperm cells we used an average excitation laser power at the objective of $20 \mathrm{~mW}$ (vs. Denk $3 \mathrm{~mW}$ ). This means the potential fluorescence flux generated after TPA-with its quadratic dependence on excitation intensity - is in our case $(20 / 3)^{2}$ or about 44 times higher, which we think was an important factor contributing to the success of our experiments considering the high fluorescence flux required from the specimen in real-time imaging. The above evaluation assumed-given the great uncertainty regarding twophoton cross-sections - comparable excitation cross-sections for the Indo-1Am dye used in the present experiment and the Hoechst 33258 used by Denk. In fact, the two-photon excitation wavelength of $780 \mathrm{~nm}$-imposed on us by the available wavelength from the amplified Ti:sapphire laser system-was substantially different from the normally used 710-720 nm wavelength (Denk et al., 1995) which would provide a much greater excitation efficiency. It is remarkable that notwithstanding this mismatch sufficient fluorescence was generated from the specimen for real-time imaging within the tolerance limits of the specimen and constitutes in our opinion a good demonstration of the value of the line scan approach for this type of imaging. Perhaps also some two-photon-generated intrinsic or auto-fluorescence from the sperm may have contributed to the signal, although this is not likely considering that bleaching limited our observation period.

The key aspect for the imaging reported above is that the thermal loading of the specimen in a line system-at equal excitation power, already much lower than in a comparable point system - increases only linearly with excitation power (see Appendix B). Owing to our excitation mismatch, we had to operate close to the tolerance limits of the specimen. This is demonstrated by the fact that, at excitation powers about $50 \%$ higher than we used for the boar sperm experiment, biological damage such as cessation of movement could be observed after a few minutes of imaging, indicating that operating conditions during the real-time imaging were close to the thermal tolerance levels of the specimen. The above indicates that we would probably not have been able to realize real-time imaging of the live specimen at the excitation powers necessary without the indicated positive effects on fluorescence excitation efficiency and specimen loading generated by the combination of the low pulse repetition rate and line excitation. In other words, it indicates that with excitation at the proper wavelengths real-time imaging at very tolerable levels of specimen loading is possible using line-excitation and amplified Ti:sapphire laser pulses.

Given the fact that biological damage is a complex process, it may be that, in addition to heating, other factors as discussed by Denk et al. (1995) may have played a role. While water absorption below $1 \mu \mathrm{m}$ seems insufficient to cause significant local heating by itself, local absorption due to accumulations of dye molecules/particulate matter in a real biological specimen may lead to local heating. Such aggregations may in fact be the start of a runaway process where the subsequent absorption by the secondary products of the heating process can lead to a 'catastrophic' thermal destruction of the specimen.

We found that the addition of a confocal aperture improves the sectioning capabilities in two-photon imaging in line mode. This is important since in TPA fluorescence imaging in the line mode, the sectioning is expected to be somewhat less than in the point mode, in analogy with the relative sectioning properties in the line and point modes in confocal microscopy (Wilson \& Hewlett, 1990)

For proper observation of the sperm cells, frame repetition rates higher than the $30 \mathrm{~Hz}$ our camera system permitted would be desirable. The bilateral scanning approach in the line mode would certainly permit this (Brakenhoff \& Visscher, 1992), as one sweep of the bilateral scan mirror generates a full image. Since scan rates up to a few kilohertz are easily realizable with present galvomirror assemblies, achieving higher image repetition rates would present no problem. CCD cameras with similar image collection frequencies are available from several manufacturers. At these high image repetition rates, the limiting factor is whether sufficient fluorescence photon flux can be generated from the specimen to achieve an acceptable noise level in the images. To assess this aspect, a similar approach can be followed as that used to determine the limitations for realtime confocal imaging (Brakenhoff \& Visscher, 1994).

Starting from real-time sectioned imaging, real-time 3-D imaging can also be accomplished by integrating or summing the data from all sections of the 3-D dataset as a charge pattern in a CCD during one video frame (Brakenhoff \& Visscher, 1994). Because of the absence of out-offocus bleaching, TPA seems very suitable in comparison with confocal sectioning as a tool for such real-time 3-Dimaging.

We think that the real-time spatial imaging capabilities offered by the combination of line bilateral scanning with two-photon excitation are quite promising for the observation of 3-D live biological specimens. For such objects (see, for instance, Denk et al., 1994) the inherent sectioning properties of TPA and-owing to the long IR excitation 
wavelength-larger penetration depths combined with less specimen interaction are of substantial value. The actual IR absorption and tolerance characteristics of a specific specimen will in general determine the possibility of using this imaging method. Present experience with the observation of various biological specimens in TPA (Denk et al., 1994) seems to indicate that a sufficient range of application areas exists for the developed real-time imaging method to be of substantial interest for the future.

\section{Acknowledgments}

We thank T. Norris for the opportunity to perform pilot TPA studies with the Ti:sapphire regenerative amplifier system. This research was financially supported in part by the Stichting Technische Wetenschappen (STW), Utrecht, The Netherlands, under grant no. ANS 33.2941 and by the National Science Foundation through the Centre of Ultrashort Optical Science under STC PHY 8920108.

\section{References}

Agart, D.A., Hiraoha, Y., Shaw, P. \& Sedat, J.W. (1989) Microscopy in three dimensions. Methods Cell Biol. 30, 353-377.

Brakenhoff, van der Voort, G.J.H.T.M., van Spronsen, E.A., Linnemans, W.A.M. \& Nanninga, N. (1985) Three-dimensional chromatin distribution in neuroblastoma nuclei shown by confocal scannong laser microscopy. Nature, 317, 748-749

Brakenhoff, G.J. \& Visscher, K. (1992) Confocal imaging with bilateral scanning and array detectors. J. Microsc. 165, 139-146.

Brakenhoff, G.J. \& Visscher, K. (1993) Imaging modes for bilateral confocal scanning microscopy. J. Microsc. 171, 17-26.

Brakenhoff, G.J. \& Visscher, K. (1994) Real-time confocal microscopy. Proc. SPIE, 2184, 93-100.

Denk, W., Strickler, J.H. \& Webb, W.W. (1990) Two-photon laser scanning fluorescence microscopy. Science, 248, 73-76.

Denk, W., Delaney, K.R., Gelperin, A., Kleinfeld, D., Strowbridge, B.W., Tank, D.W. \& Yuste, R. (1994) Anatomical and functional imaging of neurons using 2-photon laser scanning microscopy. J. Neurosci. Methods, 54, 151-161.

Denk, W., Piston, D.W. \& Webb, W.W. (1995) Two-photon molecular excitation in laser-scanning microscopy. Handbook of Biological Confocal Microscopy, 2nd edn (ed. by J. B. Pawley), pp. 445-457. Plenum Press, New York.

Deitche, J., Kempe, M. \& Rudolph, W. (1994) Resolution in nonlinear laser scanning microscopy. J. Microsc. 174, 69-73.

Hallgren, R.C. \& Buchholz, C. (1992) Improved solid surface rendering with the simulated fluorescence process (SFP) algorithm. J. Microsc. 166, RP3-RP4.

Weast, R.C. (ed.) (1974) Handbook of Chemistry and Physics, 55th edn. CRC Press, Cleveland, OH.

Rhee, J.-K., Sosnowski, T.S. \& Norris, T.B. (1994) Chirped-pulse amplification of 85 -fs pulses at $250 \mathrm{kHz}$ with third order dispersion compensationby use of holographic transmission gratings. Opt. Lett. 19, 1550-1552.

Sheppard, C.J.R. \& Gu, M. (1990) Image formation in two-photon fluorescence microscopy. Optik, 86, 104-106. van der Voort, H.T.M., Brakenhoff, G.J. \& Baarslag, M.W. (1989) Three-dimensional imaging in fluorescence by confocal scanning microscopy. J. Microsc. 153, 123-132

Wilson, T. \& Sheppard, C. (1984) Theory and Practice of Scanning Optical Microscopy. Academic Press, London.

Wilson, T. \& Hewlett, S.J. (1990) Imaging in scanning microscopes with slit-shaped detectors. J. Microsc. 160, 115-139.

\section{Appendix A: the thermal relaxation time $t_{\mathrm{th}}$}

Here we present an order of magnitude calculation of this quantity. The thermal energy content $Q$ in calories of a cubic volume of water with dimension $A(\mathrm{~m})$, i.e. with a volume of $A^{3}\left(\mathrm{~m}^{3}\right)$ with a temperature difference $T_{2}-T_{1}$ with its surroundings is given by

$$
Q=A^{3} \cdot K_{\mathrm{w}} \cdot\left(T_{2}-T_{1}\right)
$$

with $K_{\mathrm{w}}$ the thermal capacity. The thermal energy $Q$ transported from a volume element $A^{3}$ over the surface area $6 A^{2}$ of the cube with an average distance $A$ to the heat sink over a temperature difference $T_{2}-T_{1}$ during time $\Delta t$ can be estimated to be approximately equal to

$$
Q=K_{\mathrm{T}} K_{\mathrm{w}} \cdot\left(T_{2}-T_{1}\right) \cdot 6 A^{2} \Delta t / A
$$

with $K_{\mathrm{T}}$ the thermal capacity. With the thermal energy content of the volume being equal to

$$
Q=A^{3} \cdot K_{\mathrm{w}} \cdot\left(T_{2}-T_{1}\right)
$$

we find, substituting (for water) $K_{\mathrm{w}}=10^{6} \mathrm{cal} \mathrm{m}^{-3}$ and $K_{\mathrm{T}}=1.5 \times 10^{-1} \mathrm{cal} \mathrm{s}^{-1} \mathrm{~m}^{-1} \mathrm{~K}^{-1}$ (Weast, 1974) that it takes $\Delta t=A^{2} \times 10^{6} \mathrm{~s}$ for the heat to dissipate. If we take the thermal relaxation time $t_{\mathrm{th}}$ equal to this $\Delta \tau$, we find that $t_{\mathrm{th}}$ $=\mathrm{A}^{2} \times 10^{6} \mathrm{~s}$ For high-NA systems at wavelengths of around $800 \mathrm{~nm}$, as is typical for two-photon excitation, the average dimension $A$ of the focal volume is about $500 \mathrm{~nm}$, resulting in thermal relaxation times of the order of $0.25 \mu \mathrm{s}$. In practical situations, owing to aberrations the focal volume may be slightly larger and not symmetric, resulting in somewhat higher $t_{\mathrm{th}}$ values. A value of $t_{\text {th }}$ of about $1 \mu \mathrm{s}$ seems not unrealistic, indicating that the optimal pulse repetition rate to minimize thermal loading should be of the order of $1 \mathrm{MHz}$ or lower.

\section{Appendix B. TPA fluorescence generation in point and line systems}

\section{Fluorescence efficiency}

We will compare the efficiency of TPA fluorescence generation and specimen loading conditions in point and line systems, with the former excited with a high repetition rate and the latter excited with a low repetition rate pulsed laser system. Operating at an average excitation TPA power $P_{\mathrm{av}}$ the TPA excitation intensity focused in an area $A$ during a pulse is

$$
E=P_{\mathrm{av}} / f_{\mathrm{r}} \cdot t_{\mathrm{p}} \cdot A\left(\mathrm{~W} \mathrm{~m}^{-2}\right)
$$


with $f_{\mathrm{r}}$ the pulse repetition frequency and $t_{\mathrm{p}}$ the pulse duration and assuming constant intensity during the pulse. With the TPA absorption proportional to the intensity squared, the fluorescence intensity $F$ generated during a pulse of duration $t_{\mathrm{p}}$ is equal to

$$
F=\delta \cdot t_{\mathrm{p}} \cdot\left(P_{\mathrm{av}} / A \cdot f_{\mathrm{r}} \cdot t_{\mathrm{p}}\right)^{2}
$$

with $\delta$ the two-photon absorption cross-section $\left(\mathrm{m}^{4} \cdot \mathrm{s}\right.$. photon $^{-1}$ ). The total amount of fluorescence $F_{\mathrm{T}}$ per second produced by $f_{\mathrm{r}}$ pulses in a focal area $A$ is then

$$
F_{\mathrm{T}}=P_{\mathrm{av}}^{2} / A^{2} \cdot t_{\mathrm{p}} \cdot f_{\mathrm{r}}
$$

If the focal area $A$ in point and line systems is $d^{2}$ and $d \cdot l$, respectively (with $d$ the focal diameter and $l$ the cursor length), we find for the ratio of the fluorescence $R_{\text {Flp }}$ produced with the same average excitation power in line with respect point systems

$$
R_{\mathrm{Flp}}=\left(d \cdot f_{\mathrm{rp}} \cdot t_{\mathrm{pr}}\right) /\left(l \cdot f_{\mathrm{rl}} \cdot t_{\mathrm{pl}}\right)
$$

with $f_{\mathrm{rp}}, f_{\mathrm{rl}}$ and $t_{\mathrm{pr}}, t_{\mathrm{pl}}$ the pulse repetition rate and the pulse duration in respective point and line systems.

Comparing our system with the point system of Denk (1991) we note that both systems used high-NA lenses; thus $d \approx 0.35 \mu \mathrm{m}$ (estimated for a TPA wavelength of $800 \mathrm{~nm}$ and high-NA $(=1 \cdot 3)$ optics $)$. With a pulse repetition rate $f_{\mathrm{p}}=8 \times 10^{7}$ (Denk) and $f_{\mathrm{l}}=2.5 \times 10^{5}$ (our case) pulses per second and a cursor length $l=120 \mu \mathrm{m}$ and noting the equal pulse duration of $t_{\mathrm{rp}}=t_{\mathrm{rl}} \approx 100 \mathrm{fs}$ in both cases we find $R_{\mathrm{Flp}}$ $\left.=\left(0.35 \times 8.10^{7}\right) / 120 \times 2 \times 5 \times 10^{5}\right) \approx 1$, indicating that both systems will produce about equal amounts of fluorescence power for equal average two-photon excitation radiation input.

\section{Specimen loading}

The effects of the TPA exciting radiation on biological specimens are not easy to assess or evaluate. Live biological specimens not only have different sensitivity but also appear to have a distinct increased radiation sensitivity to shorter wavelengths. This type of photochemical damage is connected with interactions at the molecular level, leading to molecular bond breaking etc., and in this damage regime TPA with its long wavelengths has a distinct advantage over confocal microscopy, especially as the short wavelength radiation will also induce damage in the out-of-focus areas of the specimen. While not much is known about the relative damage sensitivity associated with single- and two-photon excitation, we assume (Denk et al., 1995) that the photochemical damage inflicted in the focal region by the doubled TPA radiation is proportional to the number of excitation events and will then be similar in both types of excitation for equivalent fluorescence output.

However, owing to the low TPA cross-sections and therefore high intensities needed in two-photon excitation, the following question can be raised: if, owing to direct absorption of the TPA radiation in the focal point, might not the resulting instantaneous temperature rise be a limiting factor in its use as an imaging technique in biological specimens? It is therefore worthwhile to compare the possible temperature rises in point and line systems at equal average TPA excitation power. With a high repetition rate point system, we have that the thermal rise in $\Delta T$ is approximately determined by accumulation of the energy absorbed over the number of pulses that occur within the thermal relaxation $t_{\text {th }}$ time of the focal volume in which this energy is deposited. Thus we find for point systems that the temperature rise $\Delta T$ will be proportional to

$$
\Delta T_{\mathrm{p}} \approx P_{\mathrm{av}} \cdot t_{\mathrm{th}} \cdot f_{\mathrm{rp}} / 3 d^{3}
$$

with $3 d^{3}$ the volume of the excitation volume. The factor 3 results from the fact that the axial length of the focal volume in high-NA systems is three times larger than in the lateral one. In line systems, where the pulse repetition rates will be much lower and in practice can be chosen easily to be (much) lower than $t_{\mathrm{th}}$, the temperature rise is basically set by the energy deposited by one pulse $\left(P_{\mathrm{av}} / f_{\mathrm{rl}}\right)$ along the cursor length in a volume of $3 d^{2}$ l:

$$
\Delta T_{\mathrm{p}} \approx P_{\mathrm{av}} /\left(f_{\mathrm{rl}} \cdot 3 d^{2} l\right) .
$$

Thus for equal average TPA excitation input for a specimen we find that the ratio of the relative temperature rise $R_{\mathrm{Tlp}}$ in the focal area in the line and point systems is

$$
R_{\mathrm{Tlp}}=\Delta T_{\mathrm{l}} / \Delta T_{\mathrm{p}}=d /\left(1 \cdot f_{\mathrm{rl}} \cdot t_{\mathrm{th}}\right)
$$

which, for the point and line systems discussed above ( $d=0.3 \mu \mathrm{m}, l=120 \mu \mathrm{m}, f_{\mathrm{rl}}=250 \mathrm{kHz}$ and $t_{\mathrm{th}}$ of the order of $1 \mu \mathrm{s} ;$ Appendix A), results in values of $R_{\text {tlp }} \approx 0.03$, indicating that much higher average TPA excitation powers in line systems can be used with respect to point systems before thermal limitations become a limiting factor.

In the above we assumed that in point scanning the beam will be positioned on a pixel element for about $4 \mu$ s, i.e. substantially longer than $t_{\mathrm{th}}$. This is the case if scanning takes place by the regular beam scanning methods where galvanometer-driven mirrors will address about 500 points per line at 500 lines $^{-1}$. With faster scanning methods, for instance acoustic deflectors, beam pixel exposure times shorter than $t_{\text {th }}$ may be produced and the relative advantage of line systems with respect to point systems will be lessened. 